\title{
Review
}

\section{Cerebrovascular Accident Risk in a Population with Periodic Limb Movements of Sleep: A Preliminary Meta-Analysis}

\author{
Tzu-Chao Lin ${ }^{a}$ Bing-Yan Zeng ${ }^{b}$ Yen-Wen Chen ${ }^{c}$ Meng-Ni Wu ${ }^{a, d}$ \\ Tien-Yu Chen ${ }^{e, f}$ Pao-Yen Ling, h Ching-Kuan Wu ${ }^{i}$ Ping-Tao Tseng ${ }^{i, j}$ \\ Chung-Yao Hsu ${ }^{\mathrm{a}, \mathrm{k}}$
}

a Department of Neurology, Kaohsiung Medical University Hospital, Kaohsiung, Taiwan; ${ }^{b}$ Department of Internal Medicine, E-Da Hospital, Kaohsiung, Taiwan; ' Prospect Clinic for Otorhinolaryngology and Neurology, Kaohsiung, Taiwan; ${ }^{d}$ Department of Master's Program in Neurology, Faculty of Medicine, Kaohsiung Medical University, Kaohsiung, Taiwan; 'Department of Psychiatry, Tri-Service General Hospital, School of Medicine, National Defense Medical Center, Taipei, Taiwan; ${ }^{\text {In }}$ stitute of Brain Science, National Yang-Ming University, Taipei, Taiwan; ${ }^{9}$ Department of Psychiatry, Kaohsiung Chang Gung Memorial Hospital and Chang Gung University College of

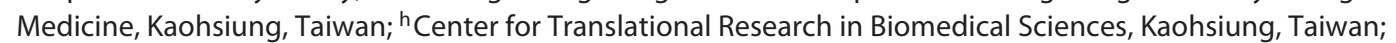
'Department of Psychiatry, Tsyr-Huey Mental Hospital, Kaohsiung, Taiwan; 'WinShine Clinics in Specialty of Psychiatry, Kaohsiung, Taiwan; ${ }^{\text {}}$ Department of Neurology, School of Medicine, College of Medicine, Kaohsiung Medical University, Kaohsiung, Taiwan

\section{Keywords}

Sleep disorders - Cerebrovascular disease · Evidence-based neurology

\section{Abstract \\ Background and Purpose: Periodic limb movements of sleep (PLMS) are usually comorbid with hypertension, tachy- cardia, and coronary arterial diseases, which are also risk fac- tors for cerebrovascular accidents (CVA). However, evidence}

T.-C.L. and B.-Y.Z. contributed equally as first author.

\section{KARGER}

() 2018 S. Karger AG, Basel

E-Mail karger@karger.com

www.karger.com/ced about the relationship between CVA and PLMS is still weak. The aim of this study was to investigate (1) the prevalence of CVA in patients with PLMS, and (2) the severity of PLMS in patients with or without CVA through a meta-analysis. Methods: The electronic databases of PubMed, Embase, ScienceDirect, ClinicalKey, Cochrane Library, ProQuest, Web of Science, and ClinicalTrials.gov were searched. The inclusion criteria were (1) articles investigating comorbidity between PLMS and CVA, and (2) clinical trials in humans. Results: This meta-analysis included (1) 9,823 patients with PLMS and 
9,416 controls from 5 studies to analyze the prevalence of CVA in PLMS, and (2) 158 patients with PLMS with CVA and 88 PLMS controls without CVA from 3 studies to analyze the severity of PLMS with and without CVA. The results showed (1) significantly higher comorbidity rates of CVA in the patients with PLMS than in the controls without PLMS (OR $1.267, p=0.019)$, and (2) higher PLM index in the patients with CVA than in the controls (Hedges' $g=0.860, p=0.001$; means difference: $4.435, p=0.016)$. Conclusions: The results revealed (1) a worse severity of PLMS in the patients with CVA, and (2) increased prevalence of CVA in the patients with PLMS. Based on our results, the patients had a higher prevalence of CVA within 8 years of a diagnosis of PLMS compared to those without PLMS by about 1.3-fold. Whether (1) patients with PLMS receiving treatment have a similar incidence of stroke to those without PLMS, and (2) secondary stroke prevention can lower the severity of PLMS or whether those with severe PLMS have a higher risk of stroke is still inconclusive. Future studies investigating the prevalence of CVA in patients with PLMS should use a follow-up period of over 8 years.

(c) 2018 S. Karger AG, Basel

\section{Introduction}

Patients with periodic limb movement in sleep (PLMS) are impacted negatively with poor sleep quality and excessive daytime sleepiness due to repeated awakenings during any sleep stage, and it can cause increased sympathetic tone [1-4]. With increasing sympathetic tone, PLMS may directly induce hypertension, tachycardia, and coronary arterial disease [4]. These comorbidities are thought to be strongly associated with cardiovascular disease (CVD) and cerebrovascular accidents (CVA) $[3,5]$. It is therefore reasonable to assume that the incidence rates of CVD and CVA would be higher in patients with PLMS. A higher prevalence of CVD has been shown in patients with PLMS in recent reviews $[1,2,6]$. However, current studies on the incidence of CVA between patients with and without PLMS have reported inconsistent results [7-9].

Parkinson's disease, multiple system atrophy, and multiple sclerosis have been associated with a worse severity of PLMS [10-12], which suggests the close association between PLMS and structural brain diseases. However, there is still lack of direct evidence from large-scale studies or conclusive evidence on the association between CVA, the most common structural brain disease, and severity of PLMS. Therefore, the aim of this study was to investigate (a) the prevalence of CVA in patients with
PLMS and control without PLMS, and (b) the severity of PLMS in patients with CVA and controls without CVA through a meta-analysis.

\section{Method and Materials}

We followed the guidelines of the meta-analysis of observational studies in epidemiology statement, which have been widely used in meta-analyses of observational trials, to perform the current meta-analysis [13] (online suppl. Table 1; for all online suppl. material, see www.karger.com/doi/10.1159/000490065). This meta-analysis (MA) was approved by the Institutional Review Board of Tri-Service General Hospital (TSGHIRB: B-10512).

Database Searches and Identification of Eligible Papers

The electronic databases of PubMed, Embase, ScienceDirect, ClinicalKey, Cochrane Library, ProQuest, Web of Science, and ClinicalTrials.gov were searched by 2 independent authors (Y.-W.C. and M.-N.W.) using the keywords "(PLM OR periodic limb movement during sleep OR PLMD OR periodic limb movement disorder) AND (cerebrovascular disease OR CVA OR infarction OR stroke)" up to March 2, 2018. Furthermore, we manually searched the reference lists of review articles or other articles related to the current topic to expand the number of potentially eligible articles [1-6, 14-19].

Two authors (T.-C.L. and B.-Y.Z.) initially screened the titles and abstracts for eligibility and generated a list of potentially eligible studies through consensus. In the next stage, 2 authors performed full-text examinations and consulted a third reviewer (P.-T.T) when there were any inconsistencies between the initial 2 authors.

\section{Inclusion and Exclusion Criteria}

The inclusion criteria were (1) published articles investigating the comorbidity between PLMS and CVA, either in the form of ischemic stroke or hemorrhagic stroke, and (2) articles that were observed clinical trials in humans. We did not set any limitations to expand the number of potentially eligible articles as far as possible.

The exclusion criteria were (1) animal studies and (2) trials investigating comorbidities other than PLMS and CVA.

Methodological Quality Appraisal

To determine the methodological quality of the included studies, we used the modified Newcastle Ottawa quality assessment scale (NOS) [20]. Scores were calculated for each study, and the studies were classified into 3 categories of quality including participant selection, comparability, and outcome. The modified NOS score ranged from zero (poor quality) to 9 (high quality).

\section{Primary Outcomes}

The primary outcome measure was the prevalence rate of (i) CVA, either in the form of ischemic stroke or hemorrhagic stroke in patients with or without PLMS, or (ii) the difference in PLM index in patients with or without CVA. The PLM index was defined as PLM occurring during sleep (PLM/hour) as recorded by polysomnography. 
Fig. 1. Flowchart of the selection strategy for the current meta-analysis. PLMS, periodic limb movement in sleep; CVA, cerebrovascular accident.

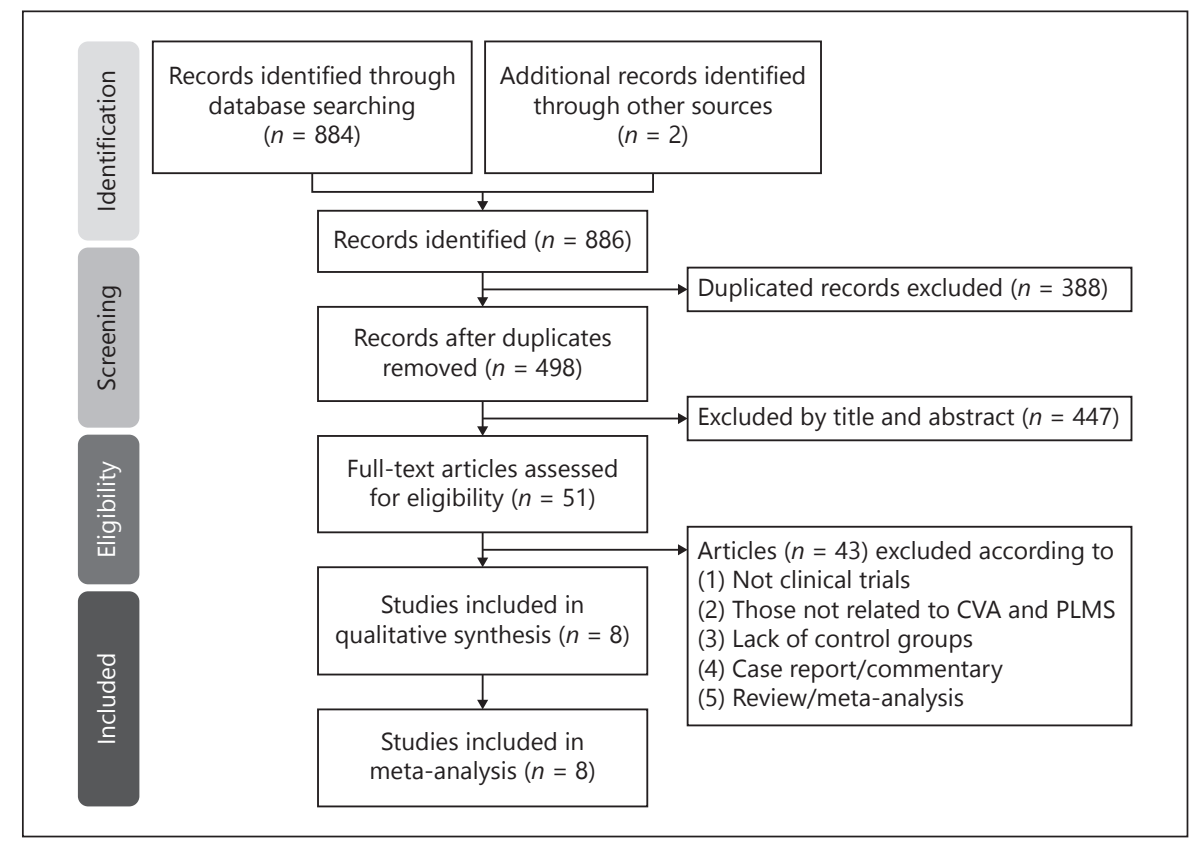

\section{Data Extraction and Management}

Two independent authors extracted data from the eligible studies into a database including a list of predetermined variables of interest, including mean age (years), gender, body mass index (BMI), apnea/hypopnea index (AHI), desaturation index, arousal index, proportion of smokers, use of benzodiazepine, sleep efficiency (\%), deep sleep phase (including N3 and N4; \%), REM sleep phase (\%), REM sleep onset latency ( $\mathrm{min})$, sleep onset latency (min), ethnicity (Caucasian, African American, Hispanic, Asian, or Native American), and modified NOS scores. When data were missed in the articles, we tried to contact the corresponding authors to request additional data.

\section{Meta-Analysis}

Under the hypothesis of potential heterogeneity among the recruited articles, we used random-effects meta-analysis models rather than fixed effect models [21] to analyze the included data using Comprehensive Meta-Analysis software, version 3 (Biostat, Englewood, NJ, USA). We assessed the effect sizes of the primary outcomes using Hedges' $g$ and 95\% CIs to examine differences in PLM index between the patients with CVA and controls, and ORs and 95\% CIs to examine the comorbidity rates of CVA in the patients with PLMS. A 2-tailed $p$ value of $<0.05$ was considered to be statistically significant.

\section{Sensitivity Test, Heterogeneity, and Publication Bias}

We assessed sensitivity using the one study removed test, which has been widely used in meta-analyses to evaluate whether or not the results of the meta-analysis resulted from any outliers within the recruited studies [22]. We used the Cochrane Q statistic with corresponding $p$ value to evaluate potential heterogeneity; we considered significant heterogeneity to be present if the $p$ value of the Q statistic was less than 0.05 [23]. We also used the $I^{2}$ statistic to indicate the proportion of heterogeneity among a study [24]. The visual inspection of funnel plots [25] and Egger's regression tests
[26] was used to evaluate potential publication bias. When significant publication bias was found, we used Duval and Tweedie's trim and fill test to adjust the effect sizes [27].

Meta-Regression and Subgroup Meta-Analyses

Due to the potential sources of heterogeneity and confounding effects, we performed meta-regression and subgroup meta-analyses to detect the potential confounding factors. Specifically, when there were at least 5 datasets, we performed meta-regression analysis using the unrestricted maximum likelihood method. The variables of interest for meta-regression were mean age (years), gender, BMI, AHI, desaturation index, arousal index, proportion of smokers, use of benzodiazepine, sleep efficiency (\%), deep sleep phase (including N3 and N4) (\%), REM sleep phase (\%), REM sleep onset latency ( $\mathrm{min}$ ), sleep onset latency ( $\mathrm{min})$, ethnicity (Caucasian, African American, Hispanic, Asian, or Native American), and modified NOS scores. The meta-analysis and subgroup meta-analyses were performed when at least 3 datasets were available [28].

\section{Results}

\section{Study Selection}

The flowchart of the current meta-analysis is shown in Figure 1. Fifty-one studies entered the full-text screen stage, of which 43 were excluded because they did not meet the inclusion criteria (reasons summarized in online suppl. Table 2). Therefore, 8 articles were eligible for the current meta-analysis (Table 1) [7-9, 29-33].

Of these 8 eligible articles, 3 provided differences in PLM index in patients with CVA and those in controls 
Table 1. Summary of characteristics of studies in current meta-analysis

\begin{tabular}{|c|c|c|c|c|c|c|c|c|}
\hline Study & $\begin{array}{l}\text { Incidence/ } \\
\text { prevalence }\end{array}$ & Comparison & Number & $\begin{array}{l}\text { PLM index/stroke } \\
\text { event }\end{array}$ & $\begin{array}{l}\text { Gender, } \\
\text { female, } \%\end{array}$ & Age, years & Trials & Country \\
\hline \multirow{2}{*}{$\begin{array}{l}\text { Coelho et al. } \\
{[29], 2010}\end{array}$} & \multirow[t]{2}{*}{ Prevalence } & PLMS + CVA & 19 & PLMI: $11.7 \pm 3.4$ & 30.0 & $63.3 \pm 1.9$ & \multirow{2}{*}{$\begin{array}{l}\text { Case-control } \\
\text { study }\end{array}$} & \multirow[t]{2}{*}{ Canada } \\
\hline & & PLMS (control) & 5 & PLMI: $1.9 \pm 0.7$ & 30.0 & $63.4 \pm 1.7$ & & \\
\hline \multirow{3}{*}{$\begin{array}{l}\text { Jiang et al. } \\
{[31], 2013}\end{array}$} & \multirow[t]{3}{*}{ Prevalence } & PLMS + VCIND & 56 & PLMI: $6.8 \pm 3.7$ & 32.0 & $63.0 \pm 2.5$ & \multirow{3}{*}{$\begin{array}{l}\text { Case-control } \\
\text { study }\end{array}$} & \multirow[t]{3}{*}{ China } \\
\hline & & PLMS + CVA & 48 & PLMI: $3.0 \pm 1.2$ & 33.0 & $61.0 \pm 5.7$ & & \\
\hline & & PLMS (control) & 48 & PLMI: $2.1 \pm 1.5$ & 31.0 & $62.0 \pm 3.5$ & & \\
\hline \multirow{2}{*}{$\begin{array}{l}\text { Benbir and } \\
\text { Karadeniz, } \\
{[30] 2012}\end{array}$} & \multirow[t]{2}{*}{ Prevalence } & PLMS + CVA & 35 & PLMI: $6.8 \pm 10.1$ & 37.1 & $68.1 \pm 9.9$ & \multirow{2}{*}{$\begin{array}{l}\text { Case-control } \\
\text { study }\end{array}$} & \multirow[t]{2}{*}{ Turkey } \\
\hline & & PLMS (control) & 35 & PLMI: $6.3 \pm 10.1$ & 68.8 & $65.7 \pm 10.1$ & & \\
\hline \multirow{3}{*}{$\begin{array}{l}\text { Chen et al. } \\
{[7], 2016}\end{array}$} & \multirow[t]{3}{*}{ Prevalence } & PLMI $\geq 15$ & 9 & Event: $6(66.7 \%)$ & 22.2 & 70.2 & \multirow{3}{*}{$\begin{array}{l}\text { Cohort } \\
\text { study }\end{array}$} & \multirow[t]{3}{*}{ Taiwan } \\
\hline & & $5 \leq \mathrm{PLMI}<15$ & 81 & Event: 6 (7.4\%) & 23.8 & 69.5 & & \\
\hline & & PLMI < 5 (control) & 46 & Event: 23 (50.0\%) & 31.7 & 59.6 & & \\
\hline \multirow{3}{*}{$\begin{array}{l}\text { Koo et al. } \\
{[8], 2011}\end{array}$} & \multirow[t]{3}{*}{ Incidence } & PLMI $\geq 30$ & 1,313 & Event: 48 (3.7\%) & 0.0 & $77.1 \pm 5.7$ & \multirow{3}{*}{$\begin{array}{l}\text { Cohort } \\
\text { study }\end{array}$} & \multirow[t]{3}{*}{ US } \\
\hline & & $5 \leq \mathrm{PLMI}<30$ & 750 & Event: 38 (5.1\%) & 0.0 & $76.1 \pm 5.5$ & & \\
\hline & & PLMI < 5 (control) & 848 & Event: $31(3.7 \%)$ & 0.0 & $75.6 \pm 5.2$ & & \\
\hline \multirow{2}{*}{$\begin{array}{l}\text { Zinchuk et al. } \\
{[32], 2018}\end{array}$} & \multirow[t]{2}{*}{ Prevalence } & PLMS + mild OSA & 119 & Event rate: $(11.3 \%)$ & 3.4 & $63.7 \pm 10.5$ & \multirow{2}{*}{$\begin{array}{l}\text { Cohort } \\
\text { study }\end{array}$} & \multirow[t]{2}{*}{ US } \\
\hline & & Non-PLMS + mild OSA & 533 & Event rate: $(7.3 \%)$ & 8.3 & $55.3 \pm 11.8$ & & \\
\hline \multirow{3}{*}{$\begin{array}{l}\text { Xie et al. } \\
{[33], 2017}\end{array}$} & \multirow[t]{3}{*}{ Prevalence } & PLMI $>30$ & 5,563 & Event: $221(4.0 \%)$ & 33.1 & (medium) 64 & \multirow{3}{*}{$\begin{array}{l}\text { Cohort } \\
\text { study }\end{array}$} & \multirow[t]{3}{*}{ US } \\
\hline & & $15 \leq$ PLMI $<30$ & 2,016 & Event: $54(2.7 \%)$ & 44.8 & 57 & & \\
\hline & & PLMI $<15$ & 7,835 & Event: $217(2.8 \%)$ & 45.7 & 55 & & \\
\hline \multirow{4}{*}{$\begin{array}{l}\text { Lindner et al. } \\
{[9], 2012}\end{array}$} & \multirow[t]{4}{*}{ Prevalence } & Transplant+ PLMI $\geq 25$ & 16 & Event: $2(12.5 \%)$ & 6.0 & $55.0 \pm 9.0$ & \multirow{4}{*}{$\begin{array}{l}\text { Cohort } \\
\text { study }\end{array}$} & \multirow[t]{4}{*}{ Hungary } \\
\hline & & Transplant + PLMI $<25$ (control) & 84 & Event: $0(0.0 \%)$ & 50.0 & $50.0 \pm 13.0$ & & \\
\hline & & Dialysis + PLMI $\geq 25$ & 16 & Event: $1(6.3 \%)$ & 31.0 & $53.0 \pm 11.0$ & & \\
\hline & & Dialysis + PLMI <25 (control) & 34 & Event: $2(5.9 \%)$ & 53.0 & $49.0 \pm 14.0$ & & \\
\hline
\end{tabular}

All the PLMS were diagnosed with AASM criteria.

AASM, American Academy of Sleep Medicine; CVA, cerebrovascular accident; PLMS, periodic limb movement during sleep; PLMI, periodic limb movement index; VCIND, vascular cognitive impairment but not meeting dementia criteria; US, United States.

(patients with CVA $=179$, mean age $=63.5$, mean female proportion $=32.9 \%$; controls without $\mathrm{CVA}=122$, mean age $=63.5$ years, mean female proportion $=30.9 \%)[29$ 31]. The other 5 articles provided the comorbidity data of CVA in patients with PLMS (patients with PLMS $=9,823$, mean age $=65.2$, mean female proportion $=28.1 \%$; controls without PLMS $=9,416$, mean age $=56.8$, mean female proportion $=39.4 \%)[7-9,32,33]$.

\section{Methodological Quality of the Included Studies}

Across the 8 studies, the average modified NOS score was 7.25 with an SD of 0.46 (online suppl. Table 3).

\section{Meta-Analysis Investigating Differences in the PLM \\ Index between the Patients with CVA and Controls without CVA}

Among the 3 eligible articles comparing differences in PLM index between the patients with CVA and controls without CVA [29-31], the meta-analysis results revealed a significantly higher PLM index in the patients with CVA than in the controls without CVA (Hedges' $g=$ $0.860,95 \%$ CI $0.359-1.361, p=0.001$; difference in means: $4.435,95 \%$ CI $0.836-8.034, p=0.016$; Fig. $2 a)$ with significant heterogeneity $(\mathrm{Q}$ value $=14.80, \mathrm{df}=3, p=0.002$; $I^{2}=79.73 \%$ ), but no publication bias was found by visual examination of the funnel plot (Fig. 2b) and via Egger's regression $(t$ value $=0.29, \mathrm{df}=2, p=0.802)$.

\section{Sensitivity Test}

The sensitivity test via one study removed revealed that the main results of the meta-analysis did not change after removing any one of the recruited studies. That is, the significance was not due to only one study.

\section{Meta-Regression}

The meta-regression procedure could not be performed on all of the clinical variables because there were fewer than 5 datasets. 


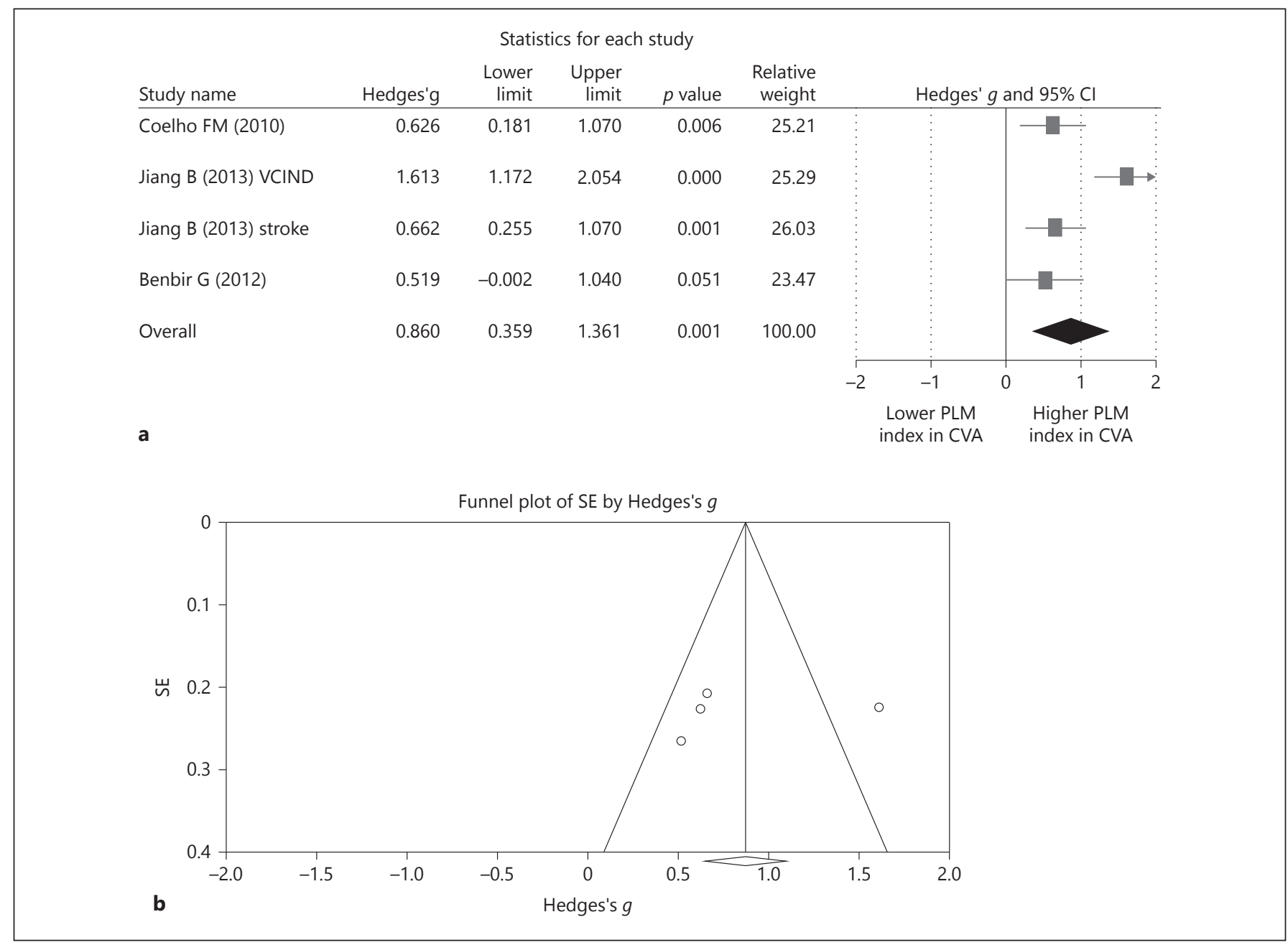

Fig. 2. a Forest plot of meta-analysis of differences in PLM index between the patients with or without CVA. b Funnel plot of metaanalysis of differences in PLM index between the patients with or without CVA. a Indicates that the PLM index was significantly higher in the patients with CVA than in those without CVA
(Hedges' $g=0.860,95 \%$ CI $0.359-1.361, p=0.001)$. b Suggests that there was no obvious publication bias detected via inspection of the funnel plot. PLM, periodic limb movement; CVA, cerebrovascular accident.

\section{Meta-Analysis Investigating the Comorbidity Rates of}

CVA in the Patients with PLMS and Controls

Among the 5 eligible articles comparing comorbidity rates of CVA in patients with PLMS and controls [7-9, $32,33]$, the meta-analysis results revealed that there was a significantly higher comorbidity rate of CVA in the patients with PLMS than in the controls without PLMS (OR 1.267, 95\% CI 1.040-1.543, $p=0.019$; Fig. 3a) without any significant heterogeneity $(\mathrm{Q}$ value $=10.385$, $\mathrm{df}=8, p=0.239 ; I^{2}=22.964 \%$, tau $\left.=0.136\right)$ or publication bias by visual examination of the funnel plot (Fig. $3 \mathrm{~b})$ and via Egger's regression $(t$ value $=0.124, \mathrm{df}=$ $7, p=0.905)$. 


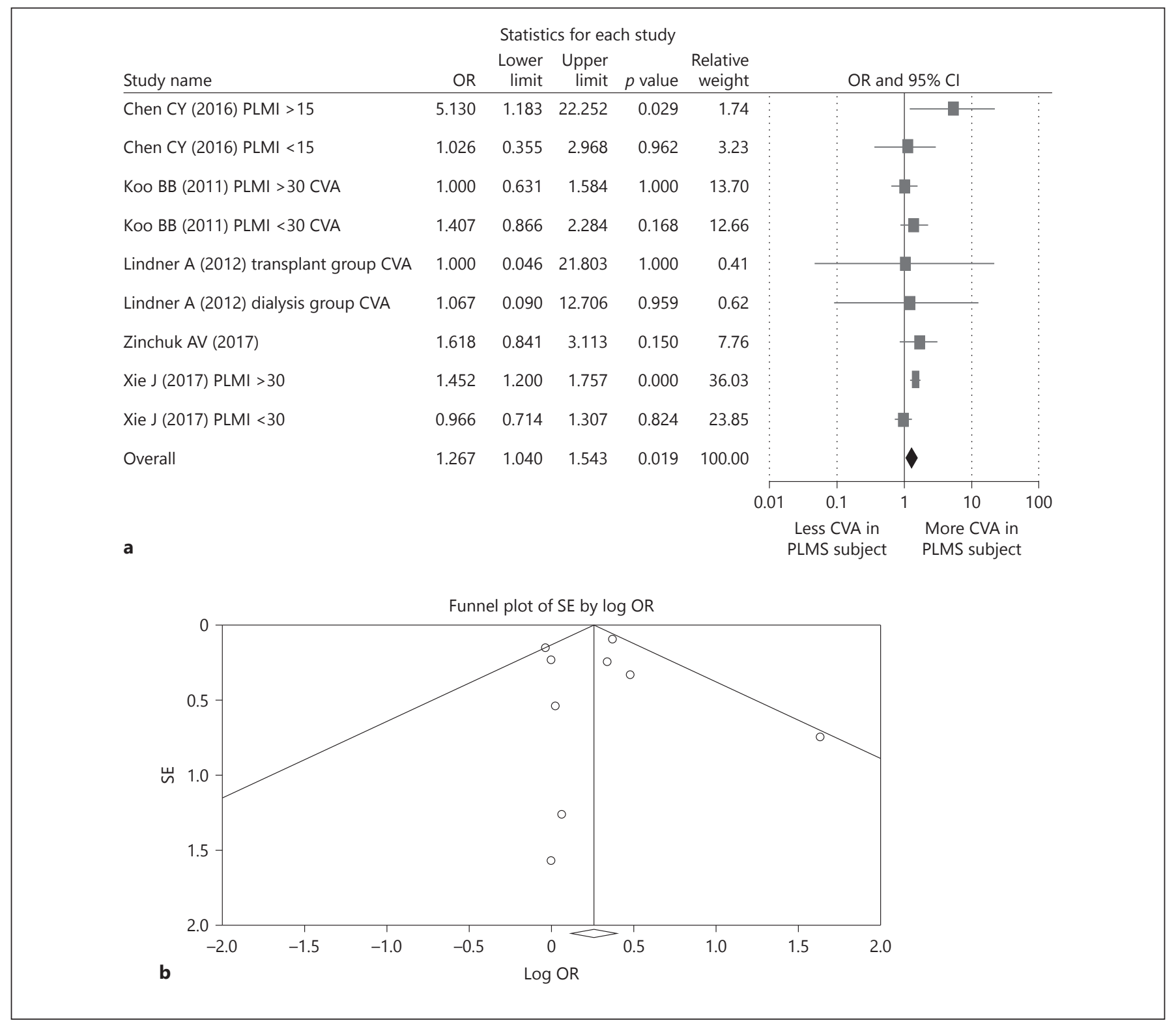

Fig. 3. a Forest plot of meta-analysis of comorbid rate of CVA in patients with or without PLMS. b Funnel plot of meta-analysis of comorbid rate of CVA in patients with or without PLMS. a Suggests that there were significantly higher comorbidity rates of CVA in the patients with PLMS than in the controls without PLMS (OR

these factors were significantly associated with the comorbidity rates of CVA mean age $(p=0.868)$, female proportion $(p=0.853)$, BMI $(p=0.884)$, mean AHI of the PLMS subjects $(p=0.219)$, and proportion of smokers in the PLMS group ( $p=0.914$; online suppl. Table 4).
$1.267,95 \%$ CI $1.040-1.543, p=0.019)$. b Indicates that there was no obvious publication bias detected via the inspection of the funnel plot. PLMS, periodic limb movement in sleep; CVA, cerebrovascular accident.

\section{Discussion}

The main results of this study are that the severity of PLMS (PLM index) was worse in the patients with CVA than in those without CVA, and that there were also significantly higher comorbidity rates of CVA in the patients with PLMS than in the controls without PLMS. The follow-up periods of the 5 analyzed studies on the preva- 
lence of CVA in the patients with PLMS were all within 8 years.

Three qualified studies reported correlations between PLMS severity and CVA [29-31] Among these 3 studies, those by Coelho et al. [29] and Jiang et al. [31] included well-randomized control and experimental groups with statistically moderate sample sizes, and Benbir et al. [30] study had a modified NOS score of 7 , indicating a high quality nonrandomized study. With regards to the significant heterogeneity among these 3 studies, the potential causes included: (a) different patient populations (geographic areas and ethnicities); and (b) different study designs and qualities. The study by Coelho et al. [29] was conducted in Canada, the study by Benbir et al. [30] was conducted in Turkey, and the study by Jiang et al. [31] was conducted in China. Therefore, even though there were no clear descriptions in any of the 3 studies, there may have been racial differences among them. In addition, the experimental groups in the studies by Benbir et al. [30] and Jiang et al. [31] were comprised of hospitalized patients with acute ischemic stroke, while the study by Coelho et al. [29] included patients attending a hospital sleep laboratory with a self-reported stroke history. The modified NOS score of Jiang et al. [31] study was 8 , which was better than the studies by Coelho et al. [29] and Benbir et al. [30] (both had an NOS score of 7). Sensitivity test showed no changes after removing any one of the recruited studies. Meta-regression was not performed due to the insufficient number of included studies. Our results are consistent with the studies by Coelho et al. [29] and Jiang et al. [31] but not with that by Benbir et al. [30], which may be due to differences in the study designs. Nevertheless, the results showed positive statistical correlations in the original articles and a strong statistical trend of Hedges' $g$ value $(p=0.051)$ in our analysis. According to the study by Jiang et al. [31], vascular cognitive impairment but not meeting dementia criteria (VCIND) seemed to be associated with a worse severity of PLMS in the patients with stroke. A possible explanation is that VCIND, compared with general stroke, is more commonly caused by structural lesions of the frontal lobe and thalamus [31], which could affect both cognitive function and sleep structure [34].

Worse PLMS severity has been reported in patients with Parkinson's disease, multiple system atrophy, and multiple sclerosis [10-12], which implicates the close relationship among PLMS and structural brain diseases despite the variety in pathophysiology. CVD, the most common structural brain disease, can cause damage to any cerebral, midbrain and brainstem area, which may then contribute to worsening the severity of PLMS as with oth- er structural brain diseases. Previous studies directly investigating relationships between PLMS and stroke have also reported higher prevalence and severity of PLMS in stroke patients, with a positive correlation between PLMS severity and white matter changes $[29,35]$. However, further information on the pathophysiology of a worse PLMS severity in stroke patients is still controversial. To date, the proposed theories have been indirect deductions, for example, a high prevalence of hypertension in stroke patients [36]; or animal models, for example, central dopamine system dysfunction [37]. Further studies on the pathophysiology of a worse PLMS in stroke patients are warranted.

With regards to the prevalence of CVA in patients with PLMS, 5 qualified studies [7-9, 32, 33] were included. With respect to randomized grouping, the patients with more severe PLM index were statistically older in the studies by Koo et al. [8], Chen et al. [7], Zinchuk et al. [32], and Xie et al. [33]. In the studies by Lindner et al. [9] and Xie et al. [33], there were significantly more male patients in the groups with more severe PLM index than in those with a less severe PLM index. Nevertheless, all 5 studies had a modified NOS score of 7 indicating mediocre randomization. The results of the studies by Chen et al. [7] (subgroup with a PLM index $>15$ ) and Xie et al. [33] (subgroup with a PLM index $>30$ ) are consistent with our results, and nonsignificant statistical trends were seen in the other subgroups in these studies.

The subgroup analysis results of patients with a PLM index $>30$ in the study by Xie et al. [33], which had a relatively large population size and long follow-up period (5 years) compared with the other subgroups, showed a statistically meaningful correlation with stroke prevalence rather than a nonsignificant trend. Possible reasons for the nonsignificant increase in stroke incidence in some study groups may include (a) low event rates leading to reduced precision and statistical power of the incidence of CVA in the patients with PLMS and (b) relatively short follow-up period and small study population in our recruited studies; this implies that with a larger sample size and longer follow-up period, the worse severity of PLMS may lead to statistically meaningful correlations with the prevalence of stroke. The results of Zinchuk et al.'s study showed a nonsignificant correlation between periodic limb movement index and CVA, which may be due to the relatively small population, despite having the longest follow-up period among all subgroups. Similarly, the subgroup with a PLN index $<30$ in Xie et al. [33] study also showed a nonsignificant correlation between periodic limb movement index and the incidence of CVA, which may have been 
due to the relatively short follow-up period, despite it having the largest study population among all subgroups.

Meanwhile, the 4 studies (Chen et al. [7], Koo et al. [8], Zinchuk et al. [32] and Lindner et al. [9]) that reported the prevalence of CVA in patients with PLMS showed a general trend that a lower PLM index was correlated with a lower incidence of CVA. This was also seen in our random-effects meta-analysis models of the studies by Chen et al. [7] and Xie et al. [33] The person-time scale of the subgroup with a PLM index $>30$ in the study by Xie et al. [33] was 22,252 person-years, compared to 39,404 person-years in the subgroup with a PLM index $<30$. Taken together, to achieve a statistically meaningful CVA comorbidity OR, the study cohort with severe PLM with a PLM index $>30$ should reach a person-time scale of 22,252 person-years; however, in the cohort with mild or moderate PLMS with a PLM index $<30$, even reaching a study person-time scale of 39,404 person-years still did not yield a statistically meaningful CVD comorbidity OR.

Previous studies have focused on the positive correlation between stroke risk factors and PLMS $[38,39]$. However, inconsistent results between the prevalence of CVA in patients with or without PLMS were reported in a previous review [40]. Through meta-analysis, we directly analyzed the relationship between CVA and PLM index. To the best of our knowledge, this review is the first meta-analysis to report a relationship between CVA and severity of PLMS, and the prevalence of CVA in patients with PLMS.

\section{Limitations}

There are some limitations to this review. First, from the analysis of comorbid CVA in the patients with PLMS, we still could not deduce whether the patients with PLMS receiving treatment had a similar incidence of stroke to those without PLMS. Second, in all eight studies included to study comorbid stroke in the patients with PLMS, we could not tell from the inclusion or exclusion criteria whether the patients took antiplatelets or anticoagulants for reasons other than stroke before study enrollment. In addition, the control of other risk factors for stroke such as strict diabetes mellitus management, anti-hypertension medication or quitting smoking were not analyzed in these 3 studies. These secondary stroke prevention treatments may have significantly decreased the incidence of stroke [41]. Finally, the heterogeneity of backgrounds, including different age, ethnicity and CVA severity may have affected the generalizability of the results. Subgroup meta-analysis was difficult to perform because of a lack of such critical information.

\section{Conclusion}

The main results of this meta-analysis suggest (a) the severity of PLMS (PLM index) is significantly worse in patients with CVA than in those without CVA, and (b) there were significantly higher comorbidity rates of CVA in the patients with PLMS than in the controls without PLMS. Based on the meta-analysis results, patients with PLMS may have a higher prevalence of CVA within 8 years of a diagnosis of PLMS compared to those without PLMS, by about 1.3-fold. With regard to further research, further studies on the potential pathophysiology between PLMS and CVA are warranted. Furthermore, future studies should also focus on whether or not treatment, which could improve the severity PLMS, would also decrease the risk of CVA in such patients.

\section{Acknowledgment}

The authors declare that there are no conflicts of interest or funding in relation to the subject of this study.

\section{Disclosure Statement}

The authors state that there are no any competing interests or funding sources in the current literature.

\section{References}

1 Alessandria M, Provini F: Periodic limb movements during sleep: a new sleep-related cardiovascular risk factor? Front Neurol 2013;4:116.

2 Cuellar NG: The effects of periodic limb movements in sleep (PLMS) on cardiovascular disease. Heart Lung 2013;42:353-360.

3 Salas RE, Rasquinha R, Gamaldo CE: All the wrong moves: a clinical review of restless legs syndrome, periodic limb movements of sleep and wake, and periodic limb movement disorder. Clin Chest Med 2010;31:383-395.

4 Somers VK, White DP, Amin R, Abraham WT, Costa F, Culebras A, Daniels S, Floras JS, Hunt CE, Olson LJ, Pickering TG, Russell R, Woo M, Young T: Sleep apnea and cardiovascular disease: An american heart association/ american college of cardiology foundation scientific statement from the american heart association council for high blood pressure research professional education committee, council on clinical cardiology, stroke council, and council on cardiovascular nursing. J Am Coll Cardiol 2008;52:686-717.

5 Marquez-Romero JM, Morales-Ramirez M, Arauz A: Non-breathing-related sleep disorders following stroke. Neurologia 2014; 29:511-516. 
6 Nannapaneni S, Ramar K: Periodic limb movements during sleep and their effect on the cardiovascular system: is there a final answer? Sleep Med 2014;15:379-384.

7 Chen CY, Yu CC, Chen CL: Nocturnal periodic limb movements decrease antioxidant capacity in post-stroke women. Acta Neurol Scand 2016;133:245-252.

8 Koo BB, Blackwell T, Ancoli-Israel S, Stone KL, Stefanick ML, Redline S; Osteoporotic Fractures in Men (Mros) Study Group: Association of incident cardiovascular disease with periodic limb movements during sleep in older men: outcomes of sleep disorders in older men (mros) study. Circulation 2011; 124:1223-1231.

9 Lindner A, Fornadi K, Lazar AS, Czira ME, Dunai A, Zoller R, Veber O, Szentkiralyi A, Kiss Z, Toronyi E, Mucsi I, Novak M, Molnar MZ: Periodic limb movements in sleep are associated with stroke and cardiovascular risk factors in patients with renal failure. J Sleep Res 2012;21:297-307.

10 Wetter TC, Collado-Seidel V, Pollmacher T, Yassouridis A, Trenkwalder C: Sleep and periodic leg movement patterns in drug-free patients with parkinson's disease and multiple system atrophy. Sleep 2000;23:361-367.

11 Hogl B, Stefani A: Restless legs syndrome and periodic leg movements in patients with movement disorders: specific considerations. Mov Disord 2017;32:669-681.

12 Chen JH, Liu XQ, Sun HY, Huang Y: Sleep disorders in multiple sclerosis in china: Clinical, polysomnography study, and review of the literature. J Clin Neurophysiol 2014;31:375-381.

13 Stroup DF, Berlin JA, Morton SC, Olkin I, Williamson GD, Rennie D, Moher D, Becker BJ, Sipe TA, Thacker SB: Meta-analysis of observational studies in epidemiology: A proposal for reporting. Meta-analysis of observational studies in epidemiology (moose) group. JAMA 2000;283:2008-2012.

14 Portaluppi F, Cortelli P, Buonaura GC, Smolensky MH, Fabbian F: Do restless legs syndrome (RLS) and periodic limb movements of sleep PLMS) play a role in nocturnal hypertension and increased cardiovascular risk of renally impaired patients? Chronobiol Int 2009;26:1206-1221.

15 Walters AS, Rye DB: Review of the relationship of restless legs syndrome and periodic limb movements in sleep to hypertension, heart disease, and stroke. Sleep 2009;32:589-597.

16 Hermann DM, Bassetti CL: Sleep-related breathing and sleep-wake disturbances in ischemic stroke. Neurology 2009;73:1313-1322.

17 Aurora RN, Kristo DA, Bista SR, Rowley JA, Zak RS, Casey KR, Lamm CI, Tracy SL,
Rosenberg RS; American Academy of Sleep Medicine: The treatment of restless legs syndrome and periodic limb movement disorder in adults - an update for 2012: practice parameters with an evidence-based systematic review and meta-analyses: an American academy of sleep medicine clinical practice guideline. Sleep 2012;35:1039-1062.

18 Koo DL, Nam H, Thomas RJ, Yun CH: Sleep disturbances as a risk factor for stroke. J Stroke 2018;20:12-32.

19 Katsanos AH, Kosmidou M, Konitsiotis S, Tsivgoulis G, Fiolaki A, Kyritsis AP, Giannopoulos S: Restless legs syndrome and cerebrovascular/cardiovascular events: Systematic review and meta-analysis. Acta Neurol Scand 2018;137:142-148.

20 Wells GA, Shea B, O'Connel D, Peterson J, Welch V, Losos M, Tugwell P: The newcastleottawa scale (NOS) for assessing the quailty of nonrandomised studies in metaanalyses; in Wells GA (ed): Clinical Epidemiology Program. Canada, 2009.

21 Borenstein M, Hedges LV, Higgins JP, Rothstein HR: A basic introduction to fixed-effect and random-effects models for meta-analysis. Res Synth Methods 2010;1:97-111.

22 Tobias A: Assessing the influence of a single study in meta-analysis. Stata Tech Bull 1999; 47:15-17.

23 Higgins JP, Thompson SG: Quantifying heterogeneity in a meta-analysis. Stat Med 2002; 21:1539-1558.

24 Borenstein M, Higgins JP, Hedges LV, Rothstein HR: Basics of meta-analysis: I2 is not an absolute measure of heterogeneity. Res Synth Methods 2017;8:5-18.

25 Higgins JP, Green S: 10.4.3.1 recommendations on testing for funnel plot asymmetry; in Higgins JP, Green S (eds): Cochrane Handbook for Systematic Reviews of Interventions. Cochrane Library, 2011. http://handbook-5-1. cochrane.org/chapter_10/10_4_3_1_ recommendations_on_testing_for_funnel_ plot_asymmetry.htm.

26 Egger M, Davey Smith G, Schneider M, Minder C: Bias in meta-analysis detected by a simple, graphical test. BMJ 1997;315:629634.

27 Duval S, Tweedie R: Trim and fill: a simple funnel-plot-based method of testing and adjusting for publication bias in meta-analysis. Biometrics 2000;56:455-463.

28 Davey J, Turner RM, Clarke MJ, Higgins JP: Characteristics of meta-analyses and their component studies in the cochrane database of systematic reviews: a cross-sectional, descriptive analysis. BMC Med Res Method 2011;11:160.
29 Coelho FM, Georgsson H, Narayansingh M, Swartz RH, Murray BJ: Higher prevalence of periodic limb movements of sleep in patients with history of stroke. J Clin Sleep Med 2010; 6:428-430.

30 Benbir G, Karadeniz D: Periodic leg movements in sleep in patients with supratentorial cerebral infarction. Acta Neurol Belg 2012; 112:27-32.

31 Jiang B, Ding C, Yao G, Yao C, Zhang Y, Ge J, Qiu E, Elia M, Ferri R: Polysomnographic abnormalities in patients with vascular cognitive impairment-no dementia. Sleep Med 2013;14:1071-1075.

32 Zinchuk AV, Jeon S, Koo BB, Yan X, Bravata DM, Qin L, Selim BJ, Strohl KP, Redeker NS, Concato J, Yaggi HK: Polysomnographic phenotypes and their cardiovascular implications in obstructive sleep apnoea. Thorax 2018;73:472-480.

33 Xie J, Chahal CAA, Covassin N, Schulte PJ Singh P, Srivali N, Somers VK, Caples SM: Periodic limb movements of sleep are associated with an increased prevalence of atrial fibrillation in patients with mild sleep-disordered breathing. Int J Cardiol 2017;241:200204.

34 Schwartz JR, Roth T: Neurophysiology of sleep and wakefulness: Basic science and clinical implications. Curr Neuropharmacol 2008;6:367-378.

35 Boulos MI, Murray BJ, Muir RT, Gao F, Szilagyi GM, Huroy M, Kiss A, Walters AS, Black SE, Lim AS, Swartz RH: Periodic limb movements and white matter hyperintensities in first-ever minor stroke or high-risk transient ischemic attack. Sleep 2017;40.

36 Gadodia G, Rangaraju S, Raza SA, Razzak A, Marmarchi L, Davis B, Henriquez L, Trotti LM, Rye D, Nahab F: Frequency of and factors associated with obstructive sleep apnea and periodic limb movements in stroke and tia patients. Neurologist 2018;23:67-70.

37 Clemens S, Rye D, Hochman S: Restless legs syndrome: revisiting the dopamine hypothesis from the spinal cord perspective. Neurology 2006;67:125-130.

38 Winkelman JW: The evoked heart rate response to periodic leg movements of sleep. Sleep 1999;22:575-580.

39 Siddiqui F, Strus J, Ming X, Lee IA, Chokroverty S, Walters AS: Rise of blood pressure with periodic limb movements in sleep and wakefulness. Clin Neurophysiol 2007;118: 1923-1930.

40 Mims KN, Kirsch D: Sleep and stroke. Sleep Med Clin 2016;11:39-51.

41 Isabel C, Calvet D, Mas JL: Stroke prevention. Presse Med 2016;45:e457-e471. 